\title{
Preface to Special Issue Dedicated to the 60th Birthday of Gregory Gutin
}

\author{
Fedor V. Fomin ${ }^{1}$. Saket Saurabh ${ }^{1}$
}

Published online: 12 February 2018

(C) Springer Science+Business Media, LLC, part of Springer Nature 2018

This special issue in Algorithmica is dedicated to Professor Gregory Gutin on the occasion of his 60th birthday and in recognition of his outstanding contributions to Discrete Mathematics and Theoretical Computer Science. Gregory Gutin is currently Professor at Department of Computer Science, Royal Holloway, University of London.

Gregory was born in 1957 in Novozybkov, Russia, when his parents were undergraduate students studying Mathematics and Physics. He received his M.Sc. in Mathematics in 1979 from Gomel State University, Belarus. After graduation, he had a number of different jobs in high schools and research institutes of Belarus. In 1990 he immigrated to Israel and started there his Ph.D. study under Professor Noga Alon at the School of Mathematics, Tel Aviv University, Israel. He received his Ph.D. with distinction in 1993. Between 1993 and 1996 he held visiting positions in the Department of Mathematics and Computer Science, Odense University, Denmark and then became a lecturer at the Department of Mathematics, Brunel University, UK. Since 2000, Gregory has worked at Royal Holloway. He is married and has three children and a grandchild.

Professor Gregory Gutin is a world-leading researcher in Discrete Mathematics, Optimization and Theoretical Computer Science. He is particularly known for his outstanding contributions in Directed Graph Theory and Algorithms.

Directed Graph Theory Gregory's standing in the area was established very early on in his career: his first results were found to be striking by the world-leading Danish mathematician Carsten Thomassen and in 1996, barely three years after his Ph.D., he

\footnotetext{
$凶$ Fedor V. Fomin

fomin@ii.uib.no

http://www.ii.uib.no/ fomin/

Saket Saurabh

http://www.ii.uib.no/ saket/

1 Department of Informatics, University of Bergen, Bergen, Norway
} 
was awarded the prestigious Kirkman Medal of the International Institute of Combinatorics and Applications. Throughout his career, Gregory has published more than 80 papers in this area alone, several of which solved open problems or developed new approaches. His influential monograph Digraphs: Theory, Algorithms and Applications has more than 2100 citations and was translated into Chinese in 2009.

Parameterized Algorithms and Complexity With more than 40 papers on the topic, Gregory is a key researcher in this area. His contributions to this area encompass many topics including parameterized algorithms and kernelization. He is responsible for solving more than 15 open problems, including the famous Rural Postperson Problem, a generalization of the Chinese Postperson Problem where we are required to find a shortest closed tour covering given edges in a graph. The complexity of this problem was a more than thirty years open [...] question with significant practical relevance (M. Sorge et al., J. Discrete Algorithms, 2012): The algorithm proposed by Gregory and his co-authors is elegant and highly efficient when the number of given edges is small, which is the case in several practical applications. His development of "above guarantee" kernelization, especially the uses of advance algebraic and probabilistic tools, advanced the area drastically.

Access Control in Information Security Gregory received best paper awards two years in a row (in 2015 and 2016) at the top conference in access control, SACMAT. These (and other) publications of his have introduced and studied a simple, yet powerful, notion of a user-independent constraint, which has led to theoretical and practical algorithms that are acknowledged to be a major breakthrough for the Workflow Satisfiability Problem. In particular, the practical algorithms significantly outperformed existing approaches based on commercial SAT and mixed integer programming solvers.

Optimization Here Gregory is well-known for his research on the fundamental Traveling Salesman Problem. He co-edited (with A.P. Punnen) the book Traveling Salesman Problem and its Variations, Springer, in 2002.

In 2014 Gregory received Royal Society Wolfson Research Merit Award and in 2017 he was elected to Academia Europaea.

The idea of this special issue came to life in January 2017, during the conference at Royal Holloway devoted to Gregory's 60th birthday, where this picture was taken. 


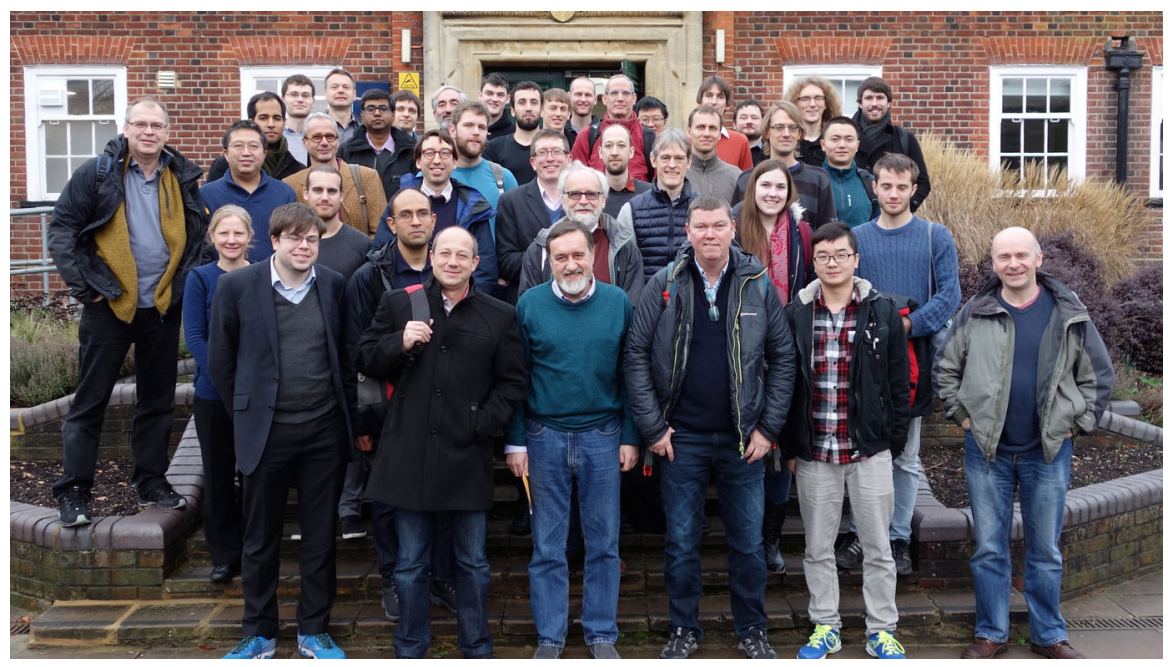

In the open call for papers the manuscripts were solicited in areas of Discrete Mathematics and Theoretical Computer Science, with special emphasis on the areas close to Professor Gregory Gutin broad research interests, including parameterized algorithms, graph algorithms, social choice theory and information security. All submissions passed through the usual for Algorithmica rigorous reviewing process. Out of 11 submissions, we selected the following papers.

- Clustering with Lower-Bounded Sizes: A General Graph-Theoretic Framework by Faisal N. Abu-Khzam, Cristina Bazgan, Katrin Casel, and Henning Fernau

- Stable Matching Games: Manipulation via Subgraph Isomorphism by Sushmita Gupta and Sanjukta Roy

- Linear Kernels and Linear-Time Algorithms for Finding Large Cuts by Michael Etscheid and Matthias Mnich

- Fréchet Distance Between a Line and Avatar Point Set by Aritra Banik, Fahad Panolan, Venkatesh Raman, and Vibha Sahlot

- Dynamic Parameterized Problems by R. Krithika, Abhishek Sahu, and Prafullkumar Tale

- Complexity of Token Swapping and its Variants by Édouard Bonnet Tillmann Miltzow, and Paweł Rzążewski

We are delighted to dedicate this special issue of Algorithmica to Prof. Gregory Gutin, an outstanding scientist and a true friend, in celebration of his 60th birthday. We wish Gregory many fruitful happy years full of exciting research!

\section{Guest Editors}

Fedor V. Fomin, http://www.ii.uib.no/ fomin/

Saket Saurabh, http://www.ii.uib.no/ saket/ 